\title{
Radical nephroureterectomy for pathologic T4 upper tract urothelial cancer: can oncologic outcomes be improved with multimodality therapy?
}

Ramy F. Youssef, Yair Lotan, Arthur I. Sagalowsky, Shahrokh F. Shariat, Christopher G. Wood, Jay D. Raman, Cord Langner, Richard Zigeuner, Marco Roscigno, Francesco Montorsi, Christian Bolenz, Wassim Kassouf, Vitaly Margulis

UT Southwestern Medical Center (RFY, YL, AIS, VM), Dallas, TX; Cornell University (SFS), New York, NY; UT M.D. Anderson Cancer Center (CGW), Houston, TX; Penn State Milton S. Hershey Medical Center (JDR), Hershey, PA, USA; Medical University of Graz (CL, RZ), Graz, Austria; Vita-Salute University (MR, FM), Milan, Italy; Mannheim Medical Center, University of Heidelberg (CB), Mannheim, Germany and McGill University, Montreal (WK), Quebec, Canada

\section{ABSTRACT}

Purpose: To report the outcomes of patients with pathologic T4 UTUC and investigate the potential impact of peri-operative chemotherapy combined with radical nephroureterectomy (RNU) and regional lymph node dissection (LND) on oncologic outcomes.

Materials and Methods: Patients with pathologic T4 UTUC were identified from the cohort of 1464 patients treated with RNU at 13 academic centers between 1987 and 2007. Oncologic outcomes were stratified according to utilization of perioperative systemic chemotherapy and regional LND as an adjunct to RNU.

Results: The study included 69 patients, 42 males (61\%) with median age 73 (range 43-98). Median follow-up was 17 months (range: 6-88). Lymphovascular invasion was found in $47(68 \%)$ and regional lymph node metastases were found in 31 (45\%). Peri-operative chemotherapy was utilized in $29(42 \%)$ patients. Patients treated with peri-operative chemotherapy and RNU with LND demonstrated superior oncologic outcomes compared to those not treated by chemotherapy and/or LND during RNU (3Y-DFS: 35\% vs. $10 \% ; \mathrm{P}=0.02$ and $3 \mathrm{Y}-\mathrm{CSS}$ : $28 \%$ vs. $14 \% ; \mathrm{P}=0.08$ ). In multivariate Cox regression analysis, administration of peri-operative chemotherapy and utilization of LND during RNU was associated with lower probability of recurrence (HR: $0.4, \mathrm{P}=0.01$ ), and cancer specific mortality (HR: $0.5, \mathrm{P}=0.06$ ).

Conclusions: Pathological T4 UTUC is associated with poor prognosis. Peri-operative chemotherapy combined with aggressive surgery, including lymph node dissection, may improve oncological outcomes. Our findings support the use of aggressive multimodal treatment in patients with advanced UTUC.

\section{ARTICLE INFO}

\section{Key words:}

Carcinoma, Transitional Cell; Drug Therapy; Lymph Nodes; Lymph Node Excision

Int Braz J Urol. 2013; 39: 614-21

Submitted for publication: January 25, 2013

Accepted after revision: September 09, 2013

\section{INTRODUCTION}

Upper tract urothelial carcinoma (UTUC) is an uncommon genitourinary malignancy accoun- ting for only 5\% of urothelial cancers and 7-8\% of all renal tumors (1). Radical nephroureterectomy (RNU) with excision of an ipsilateral bladder cuff remains the gold standard treatment for 
patients with invasive UTUC. Pathological stage is the most important predictor of oncologic outcomes in UTUC with dismal survival rates despite radical surgery for patients harboring $\mathrm{T} 4$ tumors (2-4). Therefore, patients with T4 UTUC represent a challenging cohort of patients, with limited data to guide selection of optimal therapy to maximize oncological outcomes.

Experience with urothelial carcinoma of the urinary bladder (UCB) highlights that adequate lymph node dissection (LND) in conjunction with systemic chemotherapy (particularly neoadjuvant cisplatin-based regimens), comprise a fundamental part of the contemporary multi-modal therapeutic strategy. Given biologic similarities between upper and lower tract urothelial cancer, we sought to assess the potential impact of peri-operative systemic chemotherapy and LND as an adjunct to radical RNU in patients with advanced UTUC.

Utilizing multi-institutional database of patients managed with RNU, we defined associated clinico-pathological features and oncologic outcomes of patients with pathological T4 UTUC after RNU. In an exploratory and hypothesis generating analysis, we then compared outcomes according to utilization of LND and peri-operative chemotherapy.

\section{MATERIALS AND METHODS}

\section{Patient selection}

This was an institutional review board (IRB) approved study, with all participating sites providing the necessary institutional data use agreements prior to initiation of the study. A total of 13 academic centers worldwide provided data. A computerized databank was generated for data transfer. After combining the data sets, reports were generated for each variable to identify data inconsistencies and other data integrity problems. Through regular communication with all sites, resolution of all identified anomalies was achieved before analysis. Prior to the final analysis, the database was frozen and the final data set was produced for the current analysis. This study comprised 1464 patients who underwent RNU at 13 centers between 1987 and 2007. 69 patients with pathologically confirmed T4 at RNU were identi- fied and formed the cohort of interest for this study. The choice to perform LND was determined by the surgeon and the standardization of LND was impossible due to the multicenter and retrospective design of the study.

\section{Pathologic Evaluation}

All surgical specimens were processed according to standard pathologic procedures, and all slides were re-reviewed by genitourinary pathologists according to prospectively defined uniform criteria. All pathologists were blinded to clinical outcomes. Tumors were staged according to the 7th edition of the American Joint Committee on Cancer-Union International Contre le Cancer (AJCC-UICC) Tumor-Node-Metastasis (TNM) classification (5). Tumor grades were assessed according to the 1998 WHO/ISUP (International Society of Urologic Pathology) consensus classification (6). In addition, all UTUCs were evaluated for tumor location (renal pelvis vs. ureter), pattern of tumor growth (papillary vs. sessile), presence of lymphovascular invasion (LVI), and concomitant CIS in the nephroureterectomy specimen.

\section{Surveillance Regimen}

Follow-up was performed according to institutional protocols. Patients were generally followed every three months for the first year following RNU and every six months from the second year. Follow-up consisted of a history, physical examination, routine blood work and serum chemistry studies, urinary cytology, chest radiography, cystoscopy, and radiographic evaluation of the contralateral upper urinary tract. Elective bone scans, chest computerized tomography (CT), or magnetic resonance imaging were performed when clinically indicated.

\section{Outcome Evaluation and Statistical Analysis}

Disease free survival (DFS) and cancer specific survival (CSS) were estimated using the Kaplan-Meier survival analysis. Disease recurrence was defined as local failure in the nephroureterectomy bed, regional lymph nodes (LN), or distant metastasis after RNU. The period of DFS was defined as the time between the date of RNU and the development of local recurrence or distant me- 
tastasis. Censored survival values represent patients who were alive without clinical evidence of disease at the last follow-up. Cause of death was determined by the treating physicians, by chart review corroborated by death certificates, or by death certificates alone. The period of CSS was defined as the time between the date of RNU and death due to cancer. Outcomes of patients who received peri-operative chemotherapy and RNU including LND (group 1) were compared to other patients who either did not receive any chemotherapy and/or did not undergo LND during surgery (group 2). Statistical differences were evaluated by the log rank test. All reported P values are 2-sided and significance was set at $<0.05$. Multivariate Cox Regression analyses were performed to determine the independent predictors of disease recurrence and cancer specific mortality after RNU. All statistical tests were performed with SPSS version 19.0.

\section{RESULTS}

\section{Clinico-pathological characteristics}

The study included 69 patients, 42 males (61\%) and 27 females (39\%), with median age of 73 (range 43-98). Table-1 describes clinico-pathological characteristics of the entire cohort and shows that there were no significant differences between the two groups. All tumors were high grade. LND was performed in 37 (54\%) and 31 (45\%) had LN involvement. The mean number of removed and positive LNs were 3 and 1 respectively (range 0-20 and 0-8, respectively). Systemic peri-operative chemotherapy was used in 29 $(42 \%)$ patients; $6(9 \%)$ received neoadjuvant and 23 (33\%) received adjuvant chemotherapy. Chemotherapy regimens consisted of MVAC in 60\%, Gemcitabine/Cisplatin in 20\% and other/unknown regimens in the remaining patients. Patients received an average of 3 cycles of chemotherapy (range 1 to 6).

\section{Oncological outcomes}

Median follow-up was 17 months (range: 6-88). The 3 year-DFS and CSS for the entire cohort were $18 \%$ and 26\%, respectively. The median time to recurrence and death due to cancer were 4 and 14 months, respectively. Kaplan- Meier survival analyses showed significant difference in DFS rates between patients who received both peri-operative chemotherapy and LND during RNU and the rest of the cohort (3Y-DFS: $35 \%$ vs. $10 \%$ with $\mathrm{P}=0.02$ ) (Figure-1); and a difference approaching significance in CSS (3Y-CSS: $28 \%$ vs. $14 \%$ with $\mathrm{P}=0.08$ ) (Figure-2). Median time to recurrence and death was 8 and 16.5 months, respectively, in patients who received both peri-operative chemotherapy and LND during RNU vs. 3.5 and 13 months in all others.

In multivariate Cox regression analyses (Table-2), multimodal treatment including peri-operative chemotherapy and LND during RNU was independently associated with lower probability of recurrence (HR: $0.4, \mathrm{P}=0.01$ ), and was the only factor approaching significance in multivariate analysis evaluating CSS (HR: $0.5, \mathrm{P}=0.06$ ). LN involvement was associated with disease recurrence (HR 1.97, $\mathrm{p}=0.026$ ).

\section{DISCUSSION}

Despite aggressive surgery, locally advanced UTUC is often associated with poor prognosis. Pathologic stage remains the most important predictor of survival after RNU, roughly doubling the risk of disease recurrence or death at each increment of pathologic T stage (2-4). In this multicenter retrospective analysis, T4 UTUC was associated with aggressive pathological features such as LVI, tumor necrosis, sessile architecture, concomitant CIS and regional lymph node metastases $(2,3)$. The poor prognosis of T4 UTUC was confirmed with short time to systemic relapse and nearly $75 \%$ of patients dying from their disease within 3 years after RNU. Multimodal therapy with RNU, regional LND and peri-operative chemotherapy was the only management strategy associated with improved oncologic outcomes, namely lower recurrence rates and higher survival rates. The median time to recurrence and the probability of cancer specific survival were doubled in patients managed with both peri-operative systemic chemotherapy and regional LND.

The realization of potential survival advantage that can be gained from utilization of 
Table 1 - Clinico-pathological characteristics of T4 UTUC cases at RNU.

\begin{tabular}{|c|c|c|c|c|}
\hline Variables & Entire cohort N (\%) & $\begin{array}{c}\text { Chemotherapy and LND } \\
\text { during RNU (\%) }\end{array}$ & $\begin{array}{l}\text { No chemotherapy and/or } \\
\text { LND not performed (\%) }\end{array}$ & $P$ value \\
\hline Total & 69 & $22(32)$ & $47(68)$ & \\
\hline \multicolumn{5}{|l|}{ Age $(Y)$} \\
\hline Median (range) & $73(43-89)$ & $72.5(52-89)$ & $73(43-88)$ & 0.86 \\
\hline \multicolumn{5}{|l|}{ Gender } \\
\hline Male & $42(61)$ & $15(68)$ & $27(57)$ & \multirow{2}{*}{0.39} \\
\hline Female & $27(39)$ & $7(32)$ & $20(43)$ & \\
\hline \multicolumn{5}{|c|}{ ECOG performance status } \\
\hline 0 & $46(67)$ & $16(73$ & $30(64)$ & \multirow{2}{*}{0.47} \\
\hline$\geq 1$ & $23(33)$ & $6(27)$ & $17(36)$ & \\
\hline \multicolumn{5}{|l|}{ Side } \\
\hline Right & $25(36)$ & $6(27)$ & $19(40)$ & \multirow{2}{*}{0.29} \\
\hline Left & $44(64)$ & $16(73)$ & $28(60)$ & \\
\hline \multicolumn{5}{|l|}{ Necrosis } \\
\hline Absent & $24(35)$ & $7(32)$ & $17(36)$ & \multirow{2}{*}{0.72} \\
\hline Present & $45(65)$ & $15(68)$ & $30(64)$ & \\
\hline \multicolumn{5}{|l|}{ Architecture } \\
\hline Papillary & $12(17)$ & $3(14)$ & $9(19)$ & \multirow{2}{*}{0.57} \\
\hline Sessile & $57(83)$ & $19(86)$ & $38(81)$ & \\
\hline \multicolumn{5}{|c|}{ Lymphovascular invasion (LVI) } \\
\hline Absent & $22(32)$ & $4(18)$ & $18(38)$ & \multirow[b]{2}{*}{0.09} \\
\hline Present & $47(68)$ & $18(82)$ & $29(62)$ & \\
\hline
\end{tabular}

multimodal treatment of urothelial cancers has been obtained from clinical experience with advanced bladder cancer. While radical cystectomy remains the cornerstone intervention, integration of platinum based chemotherapy and thorough LND has been shown to improve oncologic outcomes $(7,8)$. Similarly, preoperative systemic chemotherapy followed by aggressive surgical consolidation with RNU has been recently suggested to improve the oncologic outcomes of UTUC patients with loco-regional nodal metastases (9). However, not all multimodal treatment approaches were found to improve survival outcomes for patients with advanced UTUC. A previous analysis from the UTUC collaboration, including 542 high risk UTUC patients (T3,4 and/or N+), could not identify a survival advantage of the use of adjuvant chemotherapy after RNU, possibly due to the underutilization of systemic chemotherapy and administration of adjuvant chemotherapy only in advanced stage of the disease (10). The underutilization of systemic chemotherapy is not surprising, 
Figure 1 - Disease free survival probabilities based on whether peri-operative chemotherapy and LND were included in the management of T4 UTUC.

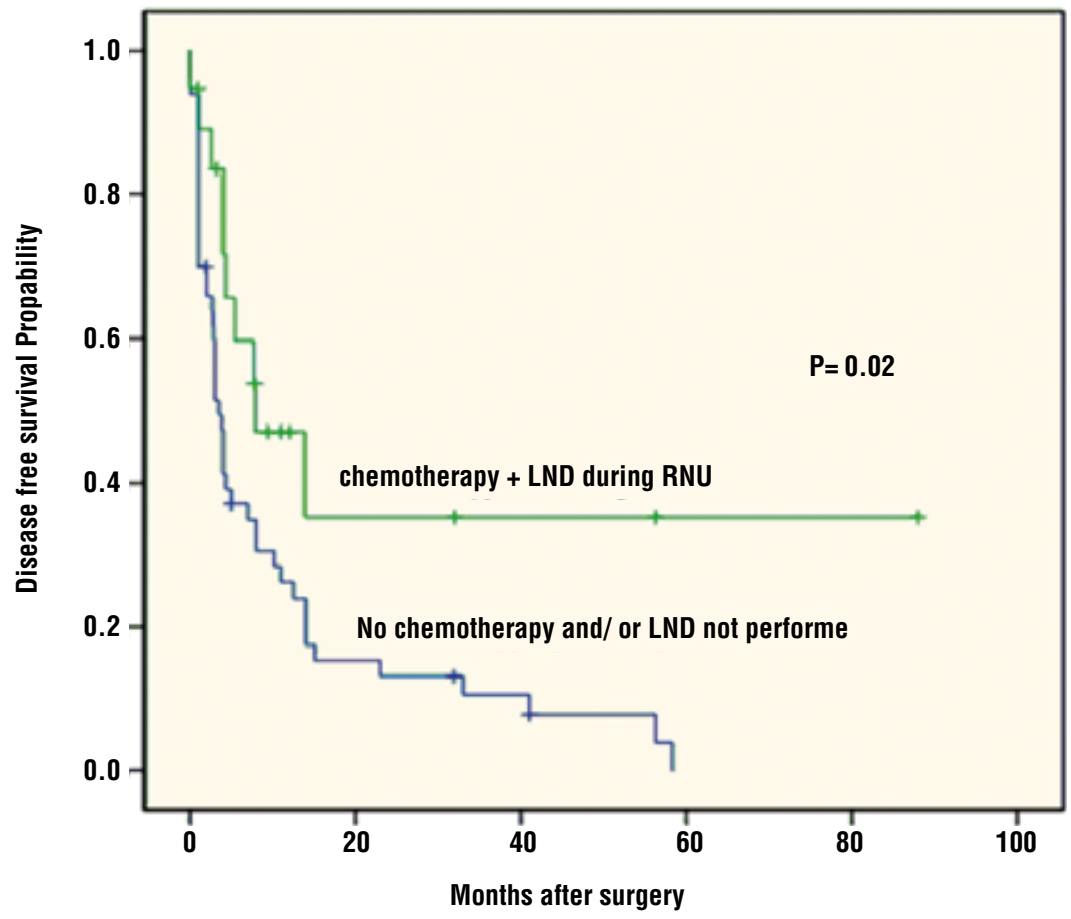

Figure 2 - Cancer specific survival probabilities based on whether peri-operative chemotherapy and LND were included in the management of T4 UTUC.

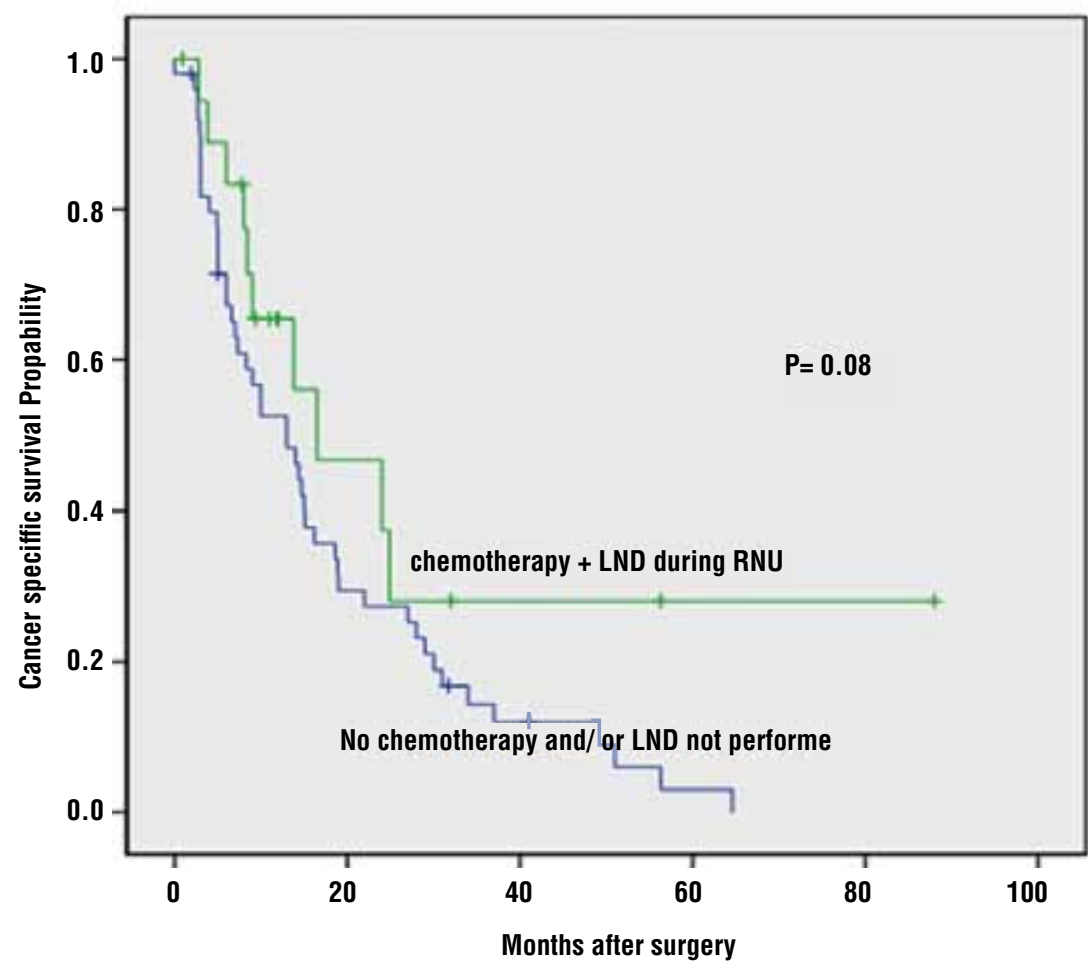


Table 2 - Multivariable Cox Regression analysis addressing disease recurrence and cancer specific mortality in 69 T4 UTUC patients treated with RNU.

\begin{tabular}{lcccccc}
\hline & & Disease recurrence & & \multicolumn{2}{c}{ Cancer-specific mortality } \\
\hline & $\mathrm{HR}$ & $95 \% \mathrm{Cl}$ & $\mathrm{P}$ & $\mathrm{HR}$ & $95 \% \mathrm{Cl}$ \\
\hline LN involvement & 1.974 & $1.083-3.596$ & 0.026 & 1.649 & $0.898-3.029$ & 0.107 \\
LVI & 1.211 & $0.630-2.328$ & 0.566 & 1.018 & $0.517-2.003$ & 0.960 \\
$\begin{array}{l}\text { Combined peri-operative chemothera- } \\
\text { py and LND }\end{array}$ & 0.397 & $0.197-0.800$ & 0.01 & 0.518 & $0.259-1.035$ \\
\hline
\end{tabular}

considering the low incidence of peri-operative chemotherapy reported in bladder cancer and the absence of randomized trials demonstrating a survival benefit in patients with UTUC (11).

There is a solid clinical rationale to utilize neoadjuvant rather than adjuvant chemotherapy for appropriately selected patients with UTUC, as the decline in renal function after nephrectomy may hinder effective chemotherapy dosing in an adjuvant setting (12-14). Moreover, a significant number of UTUC patients may already have a degree of renal insufficiency at the time of diagnosis $(13,14)$. Recent reports have shown rates of up to $14 \%$ and 53\% for complete response and down staging, after utilization of neoadjuvant chemotherapy in patients with locally advanced UTUC $(12,15)$. Neoadjuvant paradigm, however, is not devoid of potential limitations, such as overtreatment, treatment related morbidity, resulting in patient deterioration and/or disease progression during such therapy. This may explain the lower utilization of peri-operative chemotherapy, particularly in the neoadjuvant setting in our study, as only 9\% and 33\% received neoadjuvant and adjuvant chemotherapy.

Our study, similar to previous reports, has shown higher probability of LN involvement at higher T stages (16-18). Nodal status was found to be one of the most important predictors of oncologic outcomes for urothelial cancers $(4,16,19-22)$. Inadequate LND during radical surgery for urothelial cancer may underestimate the disease burden and preclude administration of adjunct systemic chemotherapy. LND might decrease overall tumor burden and allows the immune system and chemotherapeutics to target a smaller number of cancer cells, potentially with greater efficacy (21). Brausi et al. demonstrated that LND performed at the time of RNU significantly improved the survival of patients with invasive UTUC (23). Similarly, a Japanese group found that more complete LND was associated with survival benefits compared to incomplete or no LND (24). Moreover, retrospective data suggests that LND may have more significant staging and therapeutic impact on outcome in patients with locally advanced disease compared to those with localized UTUC $(16,24,25)$. However, even at the high volume academic centers included in this collaboration, LND was performed only in $40-50 \%$ of cases (25). While there is no standard, agreed upon extent or template of LND during RNU, a minimum of 8 removed LNs seemed to be the most informative cutoff with 75\% probability of finding at least 1 positive LN (22).

Several limitations of this study merit discussion. First, there are limitations inherent to the retrospective study design, which undoubtedly translate into a significant selection bias. The number of patients who presented with locally advanced disease and did not undergo surgery or progressed while receiving neoadjuvant chemotherapy is not known. Moreover, the reasons why LND was not performed during the surgery or why chemotherapy was not utilized in some patients were not included in the database. Finally, UTUC patients included in this study were managed at 
different centers by variable chemotherapy regimens and by multiple surgeons who used different surgical techniques and templates of LND. With the rarity of UTUC, a retrospective study design combining quality data from multiple high volume cancer centers may provide valuable clinical information and serve as an important guide for improving the outcomes of patients with advanced UTUC.

The integration of peri-operative chemotherapy with meticulous radical surgery, adherent to sound oncological principles including adequate LND may improve oncologic outcomes for patients with advanced UTUC.

\section{CONCLUSIONS}

Pathological T4 UTUC is associated with poor oncologic outcomes. Implementation of aggressive multimodal treatment paradigm, where peri-operative chemotherapy is integrated with meticulous radical surgery, including RNU and regional LND may improve patient outcomes.

\section{ACKNOWLEDGMENTS}

We want to thank members of the Upper Tract Urothelial Carcinoma Collaborative Group who participated in this study: Shuji Mikami, Takeo Kosaka, Masaru Isida, Keio University School of Medicine, Japan; Andrea Haitel, University of Vienna, Vienna, Austria; Roberto Bertini, Vita-Salute University, Milan, Italy; Philipp Stroebel, Maurice Stephan Michel, Universitätsklinikum Mannheim, Mannheim, Germany; Casey K. Ng, Douglas S. Scherr, Yingbei Chen, Cornell University, USA; Mario A. Fernández, Clínica Alemana de Santiago, Chile; Jeffrey Wheat, J. Stuart Wolf, Jr., University of Michigan, Michigan, USA.

\section{CONFLICT OF INTEREST}

\section{None declared.}

\section{REFERENCES}

1. Jemal A, Siegel R, Xu J, Ward E: Cancer statistics, 2010. CA Cancer J Clin. 2010; 60: 277-300.
2. Verhoest G, Shariat SF, Chromecki TF, Raman JD, Margulis V, Novara $G$, et al.: Predictive factors of recurrence and survival of upper tract urothelial carcinomas. World J Urol. 2011; 29: 495-501.

3. Chromecki TF, Bensalah K, Remzi M, Verhoest G, Cha EK, Scherr DS, et al.: Prognostic factors for upper urinary tract urothelial carcinoma. Nat Rev Urol. 2011; 8: 440-7.

4. Margulis V, Shariat SF, Matin SF, Kamat AM, Zigeuner R, Kikuchi E, et al.: Outcomes of radical nephroureterectomy: a series from the Upper Tract Urothelial Carcinoma Collaboration. Cancer. 2009; 115: 1224-33.

5. Sobin L GM, Wittekind C, editors: Renal pelvis and ureter. In: TNM classification of malignant tumours. Urological tumours. International Union Against Cancer. New York, NY. WileyBlackwell. ed. 7, Rev vol.: 2009; pp. 258-61.

6. Epstein JI, Amin MB, Reuter VR, Mostofi FK: The World Health Organization/International Society of Urological Pathology consensus classification of urothelial(transitional cell) neoplasms of the urinary bladder. Bladder Consensus Conference Committee. Am J Surg Pathol. 1998; 22: 1435-48.

7. Grossman HB, Natale RB, Tangen CM, Speights VO, Vogelzang NJ, Trump DL, et al.: Neoadjuvant chemotherapy plus cystectomy compared with cystectomy alone for locally advanced bladder cancer. N Engl J Med. 2003; 349: 859-66. Erratum in: N Engl J Med. 2003; 349: 1880.

8. Feifer AH, Taylor JM, Tarin TV, Herr HW: Maximizing cure for muscle-invasive bladder cancer: integration of surgery and chemotherapy. Eur Urol. 2011; 59: 978-84.

9. Youssef RF, Shariat SF, Lotan Y, Wood CG, Sagalowsky AI, Zigeuner $\mathrm{R}$, et al.: Upper urinary tract urothelial carcinoma with loco-regional nodal metastases: insights from the Upper Tract Urothelial Carcinoma Collaboration. BJU Int. 2011; 108: 1286-91.

10. Hellenthal NJ, Shariat SF, Margulis V, Karakiewicz PI, Roscigno $\mathrm{M}$, Bolenz $\mathrm{C}$, et al.: Adjuvant chemotherapy for high risk upper tract urothelial carcinoma: results from the Upper Tract Urothelial CarcinomaCollaboration. J Urol. 2009; 182: 900-6.

11. David KA, Milowsky MI, Ritchey J, Carroll PR, Nanus DM: Low incidence of perioperative chemotherapy for stage III bladder cancer 1998 to 2003: a report from the National CancerData Base. J Urol. 2007; 178: 451-4.

12. Matin SF, Margulis V, Kamat A, Wood CG, Grossman HB, Brown GA, et al:: Incidence of downstaging and complete remission after neoadjuvant chemotherapy for high-risk upper tract transitional cellcarcinoma. Cancer. 2010; 116: 3127-34.

13. Kaag MG, O'Malley RL, O'Malley P, Godoy G, Chen M, Smaldone $\mathrm{MC}$, et al.: Changes in renal function following nephroureterectomy may affect the use of perioperative chemotherapy. Eur Urol. 2010; 58: 581-7.

14. Alva AS, Matin SF, Lerner SP, Siefker-Radtke AO: Perioperative chemotherapy for upper tract urothelial cancer. Nat Rev Urol. 2012; 9: 266-73. 
15. Igawa M, Urakami S, Shiina $H$, Kishi $H$, Himeno $Y$, Ishibe $T$, et al.: Neoadjuvant chemotherapy for locally advanced urothelial cancer of the upper urinary tract. Urol Int. 1995; 55: 74-7.

16. Roscigno M, Shariat SF, Margulis V, Karakiewicz P, Remzi M, Kikuchi E, et al.: Impact of lymph node dissection on cancer specific survival in patients with upper tract urothelial carcinoma treated withradical nephroureterectomy. J Urol. 2009; 181: 2482-9.

17. Burger M, Shariat SF, Fritsche HM, Martinez-Salamanca Jl, Matsumoto K, Chromecki TF, et al.: No overt influence of lymphadenectomy on cancer-specific survival in organ-confined versus locally advanced upper urinary tract urothelial carcinoma undergoing radical nephroureterectomy: a retrospective international, multi-institutional study. World J Urol. 2011; 29: 465-72.

18. Lughezzani G, Jeldres C, Isbarn H, Shariat SF, Sun M, Pharand $D$, et al:: A critical appraisal of the value of lymph node dissection at nephroureterectomy for upper tract urothelial carcinoma. Urology. 2010; 75: 118-24.

19. Kondo T, Hashimoto Y, Kobayashi H, lizuka J, Nakazawa H, Ito F, et al.: Template-based lymphadenectomy in urothelial carcinoma of the upper urinary tract: impact on patient survival. Int J Urol. 2010; 17: 848-54.
20. Roscigno M, Shariat SF, Margulis V, Karakiewicz P, Remzi M, Kikuchi E, et al.: The extent of lymphadenectomy seems to be associated with better survival in patients with nonmetastatic upper-tracturothelial carcinoma: how many lymph nodes should be removed? Eur Urol. 2009; 56: 512-8.

21. Youssef RF, Raj GV: Lymphadenectomy in management of invasive bladder cancer. Int J Surg Oncol. 2011; 2011: 758189.

22. Roscigno M, Shariat SF, Freschi M, Margulis V, Karakiewizc $P$, Suardi N, et al:: Assessment of the minimum number of lymph nodes needed to detect lymph node invasion at radical nephroureterectomy inpatients with upper tract urothelial cancer. Urology. 2009; 74: 1070-4.

23. Brausi MA, Gavioli M, De Luca G, Verrini G, Peracchia G, Simonini $G$, et al.: Retroperitoneal lymph node dissection (RPLD) in conjunction with nephroureterectomy in the treatment of infiltrativetransitional cell carcinoma (TCC) of the upper urinary tract: impact on survival. Eur Urol. 2007; 52: 1414-8.

24. Kondo T, Tanabe K: The role of lymph node dissection in the management of urothelial carcinoma of the upper urinary tract. Int J Clin Oncol. 2011; 16: 170-8.

25. Roscigno M, Brausi M, Heidenreich A, Lotan Y, Margulis V, Shariat SF, et al.: Lymphadenectomy at the time of nephroureterectomy for upper tract urothelial cancer. Eur Urol. 2011; 60: 776-83.

Correspondence address: Ramy Youssef, MD Urology Division, Surgery Department Duke University Medical Center Duke South, White Zone, Rm 1576 Durham, NC 27710, USA Fax: +1 919-681-5507

E-mail: ramy.yaacoub@duke.edu 